\title{
Towards Projector-based Visualization for Computer-assisted CABG at the Open Heart
}

\author{
Christine Hartung ${ }^{1}$, Claudia Gnahm ${ }^{1}$, Stefan Sailer ${ }^{1}$, Marcel Schenderlein ${ }^{1}$, \\ Reinhard Friedl ${ }^{2}$, Martin Hoffmann ${ }^{3}$, Klaus Dietmayer ${ }^{1}$ \\ ${ }^{1}$ Institute of Measurement, Control and Microtechnology, University of Ulm \\ ${ }^{2}$ Department of Cardiac Surgery, University Hospitals of Ulm and Lübeck \\ ${ }^{3}$ Department of Diagnostic Radiology, University Hospital of Ulm \\ christine.hartung@uni-ulm.de
}

\begin{abstract}
Computer-assistance in coronary artery bypass grafting at the open heart with intraoperative navigation enables accurate placement of a bypass graft to a preoperatively planned anastomosis site. So far, navigation information were displayed on a monitor, requiring the operating surgeon to change focus from the monitor to the surgical site and vice versa during the navigation process. In this work, a mechanism is presented which is intended to enable the projection of registered preprocedural information directly onto the surface of the open heart. The method was tested on a heart phantom yielding good results.
\end{abstract}

\section{Introduction}

Open heart coronary artery bypass grafting $(\mathrm{CABG})$ is the standard treatment in advanced coronary artery diseases. For effective revascularization, optimal placement of the bypass graft is very important. Enhancing current clinical practice with a concept for computer-assistance comprising preoperative planning and intraoperative navigation helps the surgeon with the fast and accurate localization of the optimal anastomosis site for bypass grafting. For the left anterior descending coronary artery (LAD), intraoperative navigation is possible both at the beating [1] and at the arrested heart [2] yielding mean accuracies of approximately $2.5 \mathrm{~mm}$. For navigation at the beating heart, preprocedural information extracted from a multi-slice computed tomography (MSCT), i.e. the paths of the LAD vessel tree and the planned anastomosis site, are registered with intraoperative stereo video data of the open, beating heart [3], acquired with a stereo camera system mounted on a robotic arm above the patient, see fig. 1 (a). Visualization for the surgeon is currently being performed by overlaying this information on a live video stream and displaying it on a monitor, see fig. 1 (b). In consequence, the operating surgeon has to change focus from the monitor to the surgical site and vice versa during the navigation process. This can be avoided by projecting the registered planning data directly onto the surface of the open heart. In this work, a two-step calibration method is presented which aims at enabling projector-based visualization during computer-assisted CABG surgery at the open heart. 


\section{Methods}

To enable the projection of navigation information onto the surface of the heart, it has to be determined which projector pixel has to be switched on to illuminate a specific point of the surface. One possibility is to project patterns on the surface while observing it with one or more cameras. This way, point correspondences can be established between the features in the projected image and the features recorded in the camera image, and a non-linear mapping between the camera pixels and the projector pixels can be calculated. Since except for occlusions unique correspondences exists between camera pixels and surface points, this mapping also implicitely describes the correspondences between projector pixels and surface points. The standard method for generating point correspondences between camera and projector image is projecting a sequence of binary stripe patterns on the surface for binary encoding of the projector pixels. Another possibility is to project and detect a sequence of single points [4]. In both cases, a series of images has to be projected and recorded with the camera. Such methods are usually applied for using multiple projectors in the context of immersive displays [5]. In the clinical field, projectors have been proposed for displaying general information in the surgeon's field of view [6], and have already been used for navigation tasks in maxillofacial surgery [7]. While these methods work well for the described applications, they cannot directly be employed at the open heart. Novel methods have to be developed in order to consider the following additional points:

1. The heart is exposed only during the procedure, after opening the chest, and moved several times relatively to the projector before navigation. Therefore, at least part of the calibration has to be performed intraoperatively. This part must be performable fast in order to avoid prolongation of the operation.

2. The open heart is set lower than the surrounding opened chest, therefore the surface illuminated by the projector exhibits pronounced discontinuities.

3. For future usage at the beating heart, it has to be possible to perform the calibration for a specified phase point of the heart cycle. To avoid synchronizing the projector with the ECG signal over several heart cycles, it is desirable to perform the calibration process with a single projected image instead of a sequence of images.

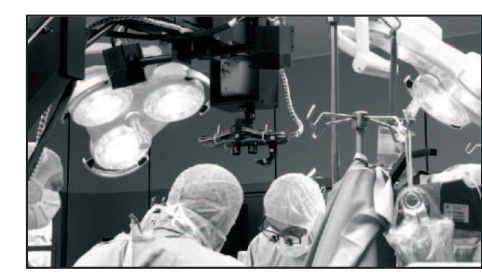

(a)

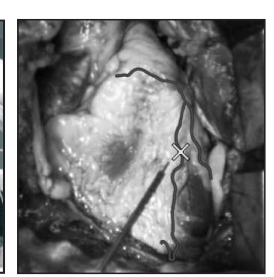

(b)

Fig. 1. (a) Stereo camera system on robotic arm, (b) registered planning data overlayed on intraoperative video images, as displayed on a monitor during surgery. 
In order to satisfy these requirements, a region of interest (ROI) based two-step method was developed for enabling the projection of planning data on the surface of the open heart during CABG procedures. It is assumed that the planning data are already registered with stereo video data of the open heart. In the first step, the paths of light rays emitted by the projector are determined. In the second step, the mapping between camera and projector for correct projection onto the surface is determined.

Step 1: A regular point grid is projected to an even surface which is positioned within the measurement volume at different distances from the stereocamera projector system. For each position, a stereo image pair is recorded. The projected points are automatically detected in the image pairs by means of a scale space approach $[8]$ and the corresponding $3 \mathrm{D}$ positions are reconstructed. Points reconstructed from different image pairs that belong to one point in the projector image, i.e. to one ray emitted by the projector are grouped and the $3 \mathrm{D}$ paths of the projector rays are reconstructed.

Step 2: The starting point of the second step of the calibration are the registered planning data, i.e. the vessel paths and a planned anastomosis site that were extracted from the MSCT data and intraoperatively registered with the coordinate system (CS) of the stereo camera system. The goal is to transform the planning data from the camera CS to the projector CS in such a way that they are projected to the correct sites on the surface of the heart. For this purpose, the point grid from calibration step 1 is projected to the heart surface and an image pair is recorded. Additionally, a disparity map of the surface is generated. The further workflow is illustrated in fig. 2. With the 2D image coordinates of the planning data in the camera CS, a ROI is defined by dilating the convex region around the vessel tree by a margin of fixed size. Within the ROI, points are automatically detected with a scale space blob detection approach [8]. Performing the calibration only for this ROI on the one hand makes the process faster, on the other hand avoids most problems with surface discontinuities. Additionally, a validity check is applied to each detected point by evaluating the disparity map for a small window around the point. If the window can be devided into two regions of significantly different disparity values by analyzing the histogram, it is assumed that the point is close to a discontinuity and it is discarded. For the valid points, 3D positions are reconstructed and compared to the paths of the projector rays from calibration step one. By determining the projector ray passing closest to each point, each valid point in the camera image is associated with one point in the projector image. Points for which the distance to the closest projector ray is too large are discarded. The

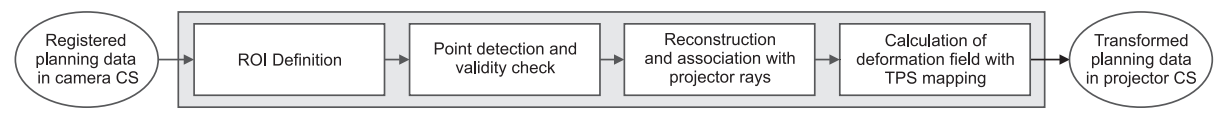

Fig. 2. Workflow of the second calibration step for projecting navigation information onto the surface of the heart. 
validity checks reduce the risk of false associations between image points and projector rays. After performing the association step, point correspondences between the image coordinate system of the camera, which is the coordinate system of the registered planning data, and the image coordinate system of the projector are available. Based on these correspondences, a smooth deformation field can be calculated using a thin-plate-spline (TPS) interpolation approach in order to describe how the planning data have to be warped to the projector image for correct projection to the surface of the heart.

A commodity projector was used in the experiment presented here. Lenses were introduced into the light path of the projector to narrow the beam width and thus reduce the size of the illuminated area, as the target area is usually smaller than $20 \mathrm{~cm}$ by $20 \mathrm{~cm}$. The method was tested on a heart phantom. To emulate the real heart in the opened chest during surgery, the heart phantom was embedded in a model of an open thorax. The ROI, i.e. the area around the vessel tree on the surface of the heart, usually does not contain surface discontinuities. However, as some of the vessels may run close to the edges between the visible part of the heart surface and the thorax, surface discontinuities might sometimes occur close to the borders of the ROI. The heart phantom and the thorax model were arranged accordingly to take this case into account.

\section{Results}

To evaluate the accuracy of the projection, an image pair of the heart phantom was acquired with the stereo camera system and the vessel tree was labeled in the left video image. Additionally a point on the LAD vessel was marked as the planned anastomosis site. The resulting $2 \mathrm{D}$ coordinates in the camera image CS simulate the registered planning data, see fig. 3 (a). These data were then transformed to the projector image coordinate system according to the method proposed above. Fig. 3 (b) shows the point grid that is projected to the heart phantom and the ROI with detected points. Fig. 3 (c) depicts the transformed planning data in the projector image and the TPS deformation field. Fig. 3 (d) shows the back projection to the heart phantom. As can be seen, the projected vessels are well aligned with the original paths of the vessels on the surface of the heart phantom, showing the viability of the proposed method. The first step of the calibration, which could be carried out before surgery in a clinical application, can be performed within in a few minutes. The second step takes less than 20 seconds and therefore could be carried out during a procedure.

\section{Discussion}

A two-step mechanism was developed which is intended to enable the projection of preprocedural information registered with intraoperative stereo video data onto the surface of the open heart during bypass grafting procedures. Evaluation of the method on a heart phantom showed accurate results. The method 
Fig. 3. (a) The vessel tree of the heart phantom is labeled in the video image, a point on the LAD is marked to simulate the planned anastomosis site, the resulting ROI is indicated. (b) The point grid is projected to the surface. Projected points are detected in the ROI (marked with circles), several points (marked with crosses) at the edge of the ROI are discarded because they are close to surface discontinuities. (c) With the determined point correspondences a TPS deformation field (represented by grid lines) from camera image to projector image is calculated and used to transform the planning data accordingly. (d) The transformed planning data are projected back to the heart phantom, showing good accordance.

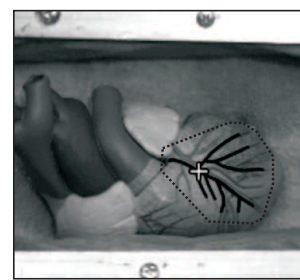

(a)

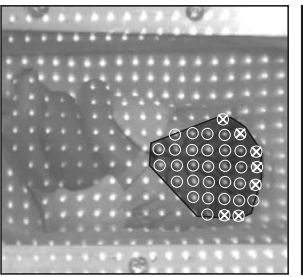

(b)

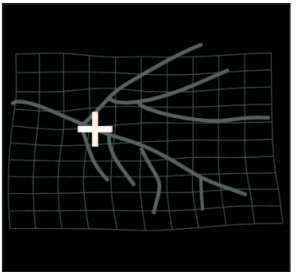

(c)

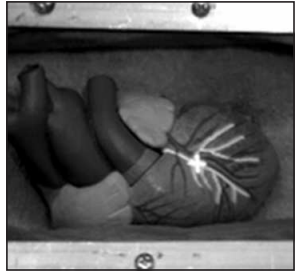

(d)

presented here differs from previous approaches to calibration methods for projection on arbitrary surfaces in the following points: (1) Instead of projecting a series of patterns to the surface, only one pattern is projected, which can be helpful for calibration at a specified heart cycle phase point. (2) A ROI is automatically determined for the calibration, which makes the process faster and avoids problems with surface discontinuities. For using the proposed mechanism in a computer-assisted open heart CABG procedure, the first calibration step should be carried out before the procedure. Since the second step takes less than 20 seconds, it could be performed intraoperatively after registration and before navigation starts without causing significant prolongation of the procedure.

\section{References}

1. Hartung, $\mathrm{C}$ et al. Computer-assisted LAD bypass grafting at the open heart. Procs SPIE. 2009.

2. Gnahm, C et al. Computer assisted navigation on the LAD during open heart CABG. Procs CURAC. 2008; p. 247-250.

3. Hartung, $\mathrm{C}$ et al. Image guidance for coronary artery bypass grafting. Int J CARS. 2008;3:505-510.

4. Raskar, R et al. Multi-Projector Displays Using Camera-Based Registration. Procs IEEE Vis. 1999; p. 161-168.

5. Brown, M et al. Camera-Based Calibration Techniques for Seamless Multiprojector Displays. IEEE Trans Vis Comput Graph. 2005;11(2):193-206.

6. Tardif, J -P et al. Projector-Based Augmented Reality in Surgery without Calibration. Procs IEEE EMBS. 2003;1:548-551.

7. Hoppe H. Projektorbasierte Erweiterte Realität in der rechnergestützten Chirurgie. GCA; 2004.

8. Lindeberg T. Scale-space Theory in Computer Vision. Kluwer Academic Publishers; 1994. 